\title{
Research on the Quality of the Three White Wine Varieties in Transylvania, Harvest of 2013-14
}

\author{
Florin Dumitru BORA ${ }^{1,3 *}$, Tiberia Ioana POP ${ }^{1 *}$, Anca Cristina BABEȘ ${ }^{1}$, Daniela POPESCU ${ }^{2}$, Maria \\ ILIESCU $^{2}$, Nastasia POP ${ }^{1 *}$ \\ ${ }^{1}$ Faculty of Horticulture, University of Agricultural Sciences and Veterinary Medicine Cluj-Napoca. \\ ${ }^{2}$ Research Station of Viticulture and Enology, Blaj, Romania. \\ ${ }^{3}$ Research Station of Viticulture and Enology, Târgu Bujor, Romania. \\ *)corresponding author, e-mail: nastpop@yahoo.com
}

BulletinUASVM Horticulture 72(2) / 2015

Print ISSN 1843-5254, Electronic ISSN 1843-5394

DOI:10.15835/buasvmcn-hort:11416

\begin{abstract}
The wine quality is based on three factors which are globally referred to as the"quality triangle": the climate and the soil (affect the quality of the grape); the type of grape (the varieties of vine-varietals); and the human factors, which include cultivation techniques, production, preservation and aging methods. The purpose of this paper is to present data regarding the composition characteristics of quality white wines. The wines were obtained from vine varieties commonly planted in Transylvania from 2013-2014, in the new climate conditions due to global warming, which increases the amount of useful temperatures during the growing season and the maturation of the grapes. The biological material used was represented by three grape varieties: 'Fetească albă', 'Fetească regală' and 'Italian Riesling', in four different areas: Baia Mare, Șimleul Silvaniei, Turulung Vii and Blaj. The values obtained for alcoholic strength and the total acidity is specific for the three analysed varieties. The wines has a quite low antioxidant protection, the average level of free $\mathrm{SO}_{2}$ being less than $50 \mathrm{mg} / \mathrm{L}(3.56-5.28 \mathrm{mg} / \mathrm{L})$ at a level of total $\mathrm{SO}_{2}$ between $44.80-52.52 \mathrm{mg} / \mathrm{L}$. Wines are microbiologically stable and this is highlighted by low volatile acidity values of $0.42 \mathrm{~g} / \mathrm{L} \mathrm{CH}_{3} \mathrm{COOH}$ in 'Italian Riesling' or $0.65 \mathrm{~g} / \mathrm{L}$ at 'Fetească albă', these values ar below of the permissible limit of $1.08 \mathrm{~g} / \mathrm{L} \mathrm{CH}_{3} \mathrm{COOH}$. Based on these results, it can be stated that the wines produced are dry, fruity, slightly acidic, yellow-green colored, and have a specific taste for each variety.
\end{abstract}

Keywords: grape varieties, physico-chemical indices, quality, white wines.

\section{INTRODUCTION}

Wine is a food product, produced exclusively by total or partial alcoholic fermentation of fresh grapes, whether or not pressed, or by must fermentation (O.I.V). From the chemical point of view, wine is a complex mixture consisting of water, ethanol, sugar, amino acids, polyphenolic compounds, anthocyanins, organic and inorganic materials (Monaci et al., 2003; Roig and Thomas 2003; Katalinic et al., 2004; Nilsson et al., 2004; Voica et al., 2009; Dalipi et al., 2015; Değirmenci Karataș et al., 2015; Marini et al., 2006; Donici et al., 2013).

Today, vines are grown throughout the world; Europe has the highest percentage (51\%) of the global area planted with vines, followed by Asia, America, and Africa (Gonçalves da Silva et al., 2008). The vineyard area in Romania has decreased since the 1990s and it currently ranks fifth in Europe after Spain, Italy, France and Portugal, and in 2013 Romania had an area of 229 000 hectares of vineyards (Lădaru et al., 2014; Tabaranu et al., 2014; Tudorache et al., 2013).

Grape and wine quality are directly influenced by several factors, natural and human: variety, soil, ecoclimatic conditions, culture, wine production process, transport and storage (Fernandez 1988; Núñez et al., 2000; Marini et al., 2006; Voica et al., 2009; Bora et al., 2014).

The physico-chemical analysis is a very important tool in knowing the composition of 
grapes; grape must and wine at different stages of evolution. Through the determination of the main physico-chemical parameters of wines, their evolution can be tracked and managed, ultimately ensuring the indices required by the standards and the internal rules in force.

The aim of this article is to determine the quality of wine from three varieties of grapes for white wines of superior quality grown in Transylvania, harvest in 2013-2014.

\section{MATERIALS AND METHODS}

There have been taken into study grape varieties for quality white wines: 'Fetească albă', 'Fetească regală' and 'Italian Riesling', grown in three areas in Transylvania: Blaj (Târnave Vineyard), Şimleul Silvaniei (Silvania Vineyard), Turulung Vii (Satu-Mare) and Baia Mare (Maramureș).

The physico-chemical wine analyzes were performed in the Oenological Laboratory of the Faculty of Horticulture, University of Agricultural Sciences and Veterinary Medicine of Cluj-Napoca, according to the methods of analysis described in the Compendium of international methods of analysis of wines and musts (OIV- last edition 2009), but also following the Romanian STAS methods.

The alcoholic strength (\% vol.) was determined using the ebulliometric method, STAS 6182/6-70, which is based on the principle of temperature difference between boiling water and ethyl alcohol $\left(78.4^{\circ} \mathrm{C}\right)$, wine being a hydroalcoholic mix.

Total acidity (titratable acidity) is defined as the total substances with acid reaction present in wine, which can be titrated with an alkaline solution in the presence of an indicator.

The total acidity of the wine was determined by titrimetric method, STAS 6182-1:2008. The principle of this method lies in the titration or neutralization of the acids from the sample to be analyzed (wine) with a sodium hydroxide solution with known normality and factor, in the presence of phenolphthalein as an indicator, after the removal of carbon dioxide.

Results were calculated using the formula: Total acidity (in tartric acid) $=0.75 \times \mathrm{n} \mathrm{x} \mathrm{f}(\mathrm{g} / \mathrm{l})$

$n=\mathrm{NaOH} 0.1 \mathrm{~N}$, $f=$ factor $=0.9963$.

The volatile acidity of wine was determined according to STAS 6182-2:2008, through the titration of volatile acids, using the water vapors, by the formula:

$$
\begin{aligned}
& \text { A (acetic acid } g / l)=0.300 \times\left(n-0.1 n^{1}-0.05 n^{2}\right) \\
& n=\mathrm{NaOH} 0.1 \mathrm{~N} \\
& n^{1}=\text { volume of iodine } 0.005 \mathrm{M} \text { used in first } \\
& n^{2}=\text { volume of iodine } 0.005 \mathrm{M} \text { used in first }
\end{aligned}
$$$$
\text { titration }(\mathrm{ml}) \text {; }
$$$$
\text { titration }(\mathrm{ml}) \text {; }
$$

The total dry extract was determined according to STAS 6182/9-80, using the direct method consisting in evaporating a volume of the wine $(50 \mathrm{ml})$ using the water bath. The total extract represents all nonvolatile matter which in specific physical conditions do not volatilize. From the chemical point of view, the matter is represented by: fixed organic acids (tartaric, malic, succinic acid, lactic acid), glycerol, 2,3 butylene glycol, sugars, tannins and dyes, nitrogen, pectin, gums, etc. The formula $(\mathrm{g} / \mathrm{l})$ :

Dry extract $=\left[\left(m_{2}-m_{1}\right) / V\right] \times 1000$

$m_{1}=$ weight of the empty capsule,

$m_{2}=$ weight of the capsule with the extract,

$V=$ volume of wine $(\mathrm{ml})$.

Nonreducing dry extract is the difference between the total extract and the total sugars.

Total sugars were determined according STAS 6182/17-81 and reducing sugars according to STAS 6182-18:2009. The principle of the method consists in treating the sample to be analyzed, which is the dealcoholized wine (by evaporation on a water bath to half volume) with a solution of basic lead acetate, in order to precipitate the protein substances, gums and mucilages and the filtration of the precipitate. Dosing of sugar was conducted using the Bertrand method.

Free and total sulphur dioxide $\left(\mathrm{SO}_{2}\right)$ was determined by iodometric method, according to STAS 6182/13:2009. Free $\mathrm{SO}_{2}$ is $\mathrm{SO}_{2}$ from wine, in a state of mineral combinations (uncommitted in reactions with organic substances). It represents approximately $30 \%$ of total $\mathrm{SO}_{2}$. The formula for free $\mathrm{SO}_{2}$ :

$\mathrm{SO}_{2}(\mathrm{mg} / \mathrm{l})=\left[\left(\mathrm{V}_{1}-\mathrm{V}_{2}\right) \times 0.64 \times 1000\right] / 50=12.8$ $\left(\mathrm{V}_{1}-\mathrm{V}_{2}\right)$

$\mathrm{V}_{1}=$ volume of iodine $0.02 \mathrm{~N}$ used in first titration (ml);

$\mathrm{V}_{2}=$ volume of iodine $0.02 \mathrm{~N}$ used in second titration (ml), which reacts with the substances with reducing nature of wine (tannins and dyes) 
$0.64=$ quantity of $\mathrm{SO}_{2}(\mathrm{mg})$ corresponding to 1 $\mathrm{ml}$ iodine $0.02 \mathrm{~N}$.

The total $\mathrm{SO}_{2}$ represents the assemble of organic forms resulted by combining $\mathrm{SO}_{2}$ with aldehydes, ketones, acids, sugars, uronic acids, oxidation products of sugars, phenolic compounds and other substances. The formula for total $\mathrm{SO}_{2}$ from wine:

Total $\mathrm{SO}_{2}(\mathrm{mg} / \mathrm{l})=(\mathrm{V} \times 0.64 \times 1000) / 50=12.8 \mathrm{~V}$

$V=$ volume of iodine $0.02 \mathrm{~N}$ used in both titrations ( $\mathrm{ml}$ );

$0.64=$ quantity of $\mathrm{SO}_{2}(\mathrm{mg})$ which corresponds to $1 \mathrm{ml}$ iodine $0.02 \mathrm{~N}$.

Statistical interpretation of results was done with the Duncan test (ARDELEAN, 1986) using version 20 of SPSS (SPPS Inc. Chicago, IL, USA). The statistical processing of results was done primarily to calculate the following statistical parameters: arithmetic mean, standard deviation, mean error by using the statistical package SPSS. Data was interpreted by analysis of variance (ANOVA), media separation was done using the Duncan test at $\mathrm{P} \leq 0.05$. The interaction between area and species was evaluated by selecting $\mathrm{P} \leq$ $0.0001, \mathrm{P} \leq 0.01$ and $\mathrm{P} \leq 0.05$ to determine the significance.

\section{RESULTS AND DISCUSSION}

By analyzing each variety, it has been noted that wines showed varying alcohol content. Differences between variants were statistically assured $(\mathrm{F}=27.85, \mathrm{p} \leq 0.000)$. In 2014 , the highest alcohol amount was recorded in 'Italian Riesling' from Blaj (13.0\% vol.) and 'Fetească albă' from Șimleul Silvaniei (12.8 \% vol.). The other varieties taken into study were statistically equal. A lower alcoholic strength was recorded by the three varieties in Baia Mare in 2013 ('Fetească albă' $10.1 \%$ vol., 'Fetească regală' $9.8 \%$ vol. and 'Italian Riesling' $10.5 \%$ vol.). Baia Mare is not an area suited for cultivation of vines, most often the obtained wine being current table wine, and in some years, it even has a lower quality due to the bioclimatic index values, which are below the lower limit 5 . In this area, the vines are grown mostly in a culture of amateurism (Tab. 1).

The year factor had the biggest influence on the alcohol degree $(F=278.301, p \leq 0.000)$ and the location factor $(F=74.66, p \leq 0.000)$, followed by the variety factor $(\mathrm{F}=31.37, \mathrm{p} \leq 0.000)$. The interaction between location $\mathrm{x}$ variety had $\mathrm{a}$ very significant influence on the alcohol degree $(\mathrm{F}=6.99, \mathrm{p} \leq 0.000)$, followed by the interaction location $\mathrm{x}$ year, which had a significantly distinct influence on the character ( $F=5.56, p=0.002)$. The interaction between the three factors (location $\mathrm{x}$ variety $x$ year) had a significant influence on this character $(\mathrm{F}=2.67, \mathrm{p}=0.025)$.

In the case of total acidity $\left(\mathrm{g} / \mathrm{L} \mathrm{C}_{4} \mathrm{H}_{6} \mathrm{O}_{6}\right)$ it can be observed that this parameter was very significantly influenced by the location factor $(\mathrm{F}=264.75, \mathrm{p} \leq 0.000)$, then the variety $(\mathrm{F}=56.24$, $\mathrm{p} \leq 0.000)$ and the year $(\mathrm{F}=29.02, \mathrm{p} \leq 0.000)$. The interaction between location $x$ variety $(\mathrm{F}=39.40$, $\mathrm{p} \leq 0.000)$, location $\mathrm{x}$ year $(\mathrm{F}=69.39, \mathrm{p} \leq 0.000)$, variety $\mathrm{x}$ year $(\mathrm{F}=28.45, \mathrm{p} \leq 0.000)$ and the interaction between the three factors had a very significant influence on this character (Tab. 1).

Regarding the volatile acidity ( $\left.\mathrm{g} / \mathrm{L} \mathrm{CH}_{3} \mathrm{COOH}\right)$, the data presented in Tab. 1 shows that this parameter has variable values for the analyzed variants. The highest values were recorded in the 'Fetească albă' wine cultivated in Turulung Vii $\left(0.65 \mathrm{~g} / \mathrm{L} \mathrm{CH}_{3} \mathrm{COOH}\right)$ and Baia Mare $(0.59 \mathrm{~g} / \mathrm{L}$ $\mathrm{CH}_{3} \mathrm{COOH}$ ). The lowest values were recorded in wines produced in Blaj area in $2014(0.32 \mathrm{~g} / \mathrm{L}$ $\mathrm{CH}_{3} \mathrm{COOH}$ 'Italian Riesling'; $0.33 \mathrm{~g} / \mathrm{L} \mathrm{CH}_{3} \mathrm{COOH}$ 'Fetească regală'), Târnave vineyard being characterized by the lowering of the acidity, if grapes remain in plantation until full maturity. Therefore, it is recommended to harvest earlier than the optimal harvest time for wines to be balanced. The differences between variants were statistically assured $(\mathrm{F}=8.12, \mathrm{p} \leq 0.000)$. The biggest influence on the volatile acidity was given by the location factor $(F=24.90, p \leq 0.000)$, followed by the location $\mathrm{x}$ variety interaction between factors $(\mathrm{F}=7.86, \mathrm{p} \leq 0.000)$ and the interaction between the three main location $\mathrm{x}$ variety $\mathrm{x}$ year had a very significant influence on this character $(\mathrm{F}=5.09$, $\mathrm{p} \leq 0.000$ ).

The higher $\mathrm{pH}$ was recorded in the wine obtained from 'Fetească regală', produced in 2013 and cultivated in Turulung Vii (3.7) and Şimleul Silvaniei (3.6). The wine obtained from the 'Italian Riesling' variety, grown in Blaj in 2014, had the lowest $\mathrm{pH}$ (3.1). Differences between variants were statistically assured $(\mathrm{F}=4.27, \mathrm{p} \leq 0.000)$. The variety factor had the greatest influence on this character $(\mathrm{F}=25.10, \mathrm{p} \leq 0.000)$. 
The highest density was recorded in 2013, in the wine made from the 'Italian Riesling' variety grown in Turulung Vii area $\left(0.9979 \mathrm{~g} / \mathrm{cm}^{3}\right)$, followed by the same variety grown in Şimleul Silvaniei area $\left(0.9980 \mathrm{~g} / \mathrm{cm}^{3}\right)$, the two versions are equal in statistical terms. The lowest density was recorded in 2013 in 'Fetească regală' from Blaj, followed by wine produced from 'Fetească albă' from the same area $\left(0.9936 \mathrm{~g} / \mathrm{cm}^{3}\right)$. Differences between variants were statistically assured $(\mathrm{F}=14.12, \quad \mathrm{p} \leq 0.000)$ (Table 1$)$. The greatest influence on the density of wine had the location factor $(\mathrm{F}=81.92, \mathrm{p} \leq 0.000)$, followed by the variety factor $(\mathrm{F}=34.29, \mathrm{p} \leq 0.000)$, while the year factor had no influence $(\mathrm{F}=0.60, \mathrm{p} \leq 0.440)$. The interaction between location $x$ variety $(F=7.34, p \leq 0000)$ had a very significant influence, while the interaction between variety $\mathrm{x}$ year had a distinct significant influence ( $\mathrm{F}=7.69, \mathrm{p}=0.001)$ (Tab. 1).

The residual sugar content $(\mathrm{g} / \mathrm{L})$ was very significantly influenced by the location factor $(\mathrm{F}=71.39, \mathrm{p} \leq 0.000)$, the variety $(\mathrm{F}=24.42$, $\mathrm{p} \leq 0.000$ ), while the year factor had a distinctly significant influence $(\mathrm{F}=5.53, \mathrm{p} \leq 0.000)$. The interactions between the location $\mathrm{x}$ variety $(\mathrm{F}=118.55, \mathrm{p} \leq 0.000)$, location $\mathrm{x}$ year $(\mathrm{F}=158.55$, $\mathrm{p} \leq 0.000)$, variety $\mathrm{x}$ year $(\mathrm{F}=38.29, \mathrm{p} \leq 0.000)$, had a very significant influence, as well as the interaction of the three factors location $\mathrm{x}$ variety $x$ year $(F=67.43, p \leq 0.000)$, had a very significant influence on this character (Tab. 2).

The highest content of total dry extract was recorded in wines from 'Italian Riesling' variety (28.7 g/L in 2013; 27.6 g/L in 2014) from Turulung Vii, followed by Şimleul Silvaniei $(28.6 \mathrm{~g} / \mathrm{L}$ in 2013; $27.9 \mathrm{~g} / \mathrm{L}$ in 2014), while the lowest rates were registered in the varieties grown in the Blaj area (18.7 g/L in 2013 and 19.5 in 2014) and Baia Mare (18.5 g/L in 2013). The differences between variants were statistically assured $(\mathrm{F}=30.88$, $\mathrm{p} \leq 0.000$ ) (Tab. 2).

The biggest influence on the non-reducing dry extract content was given by the location factor $(\mathrm{F}=88.81, \mathrm{p} \leq 0.000)$, and year $(\mathrm{F}=95.13, \mathrm{p} \leq$ $0.000)$, followed by variety $(\mathrm{F}=61.63, \mathrm{p} \leq 0000)$. The interaction of factors had a very significant influence: location $\mathrm{x}$ year $(\mathrm{F}=33.61, \mathrm{p} \leq 0.000)$, location $\mathrm{x}$ variety $(\mathrm{F}=21.80, \mathrm{p} \leq 0.000)$, and variety $\mathrm{x}$ year $(\mathrm{F}=11.19, \mathrm{p} \leq 0.000)$, while the interaction of three factors location $\mathrm{x}$ variety $\mathrm{x}$ year had a significant influence on the character $(\mathrm{F}=1.48$, $\mathrm{p}=0.204$ ).

Regarding the free sulfur dioxide content of wine, wines grown in Blaj had the highest values in 2014 (50.93 $\pm 1.94 \mathrm{~g} / \mathrm{L}$ 'Italian Riesling', $47.73 \pm 1.84 \mathrm{~g} / \mathrm{L}$ 'Fetească albă' and $35.91 \pm 1.45 \mathrm{~g} / \mathrm{L}$ 'Fetească regală') compared to wines produced in Turulung Vii in 2013 (3.56 $\pm 0.05 \mathrm{~g} / \mathrm{L}$ 'Fetească regală' and $4.52 \pm 0.07 \mathrm{~g} / \mathrm{L}$ 'Fetească albă'). This is due to the use of a smaller quantity of sulfur dioxide for clarifying and preserving the wine. The differences between variants were statistically assured ( $\mathrm{F}=530.25, \mathrm{p} \leq 0.000$ ) (Tab. 2).

The location factor had the greatest influence on sulfur dioxide $(\mathrm{F}=3074.40, \mathrm{p} \leq 0.000)$, and year factor $(\mathrm{F}=304.99, \mathrm{p} \leq 0.000)$, followed by the variety factor $(\mathrm{F}=23.29, \mathrm{p} \leq 0.000)$. The interaction of the factors had a very significant influence: location $\mathrm{x}$ year $(\mathrm{F}=232.68, \mathrm{p} \leq 0.000)$, location $\mathrm{x}$ variety $(\mathrm{F}=86.27, \mathrm{p} \leq 0.000)$, while the interaction between variety $\mathrm{x}$ year had a distinctly significant influence on this character $(F=6.47, p=0.003)$. The interaction between the three factors location $\mathrm{x}$ variety $\mathrm{x}$ year had a very significant influence $(\mathrm{F}=65.41, \mathrm{p} \leq 0.000)$.

Comparing the results of free $\mathrm{SO}_{2}$ content with the legislation, it can be seen that all produced wines have a much lower content than the one required by law, therefore the wines can be consumed/preserved.

The highest amount of total $\mathrm{SO}_{2}$ was registered in the wine produced in Blaj area $(186.76 \pm 3.83$ mg/L in 'Fetească regală' and 169.72 \pm 4.54 mg/L in 'Fetească albă' in 2013). The wine made from 'Italian Riesling' variety grown in Blaj in both years of study, had high levels of total $\mathrm{SO}_{2}$ concentration $(176.17 \pm 1.52 \mathrm{mg} / \mathrm{L}$ in 2013 and $170.65 \pm 3.47 \mathrm{mg} / \mathrm{L}$ in 2014). At the opposite end was the wine from 'Fetească regală' in Simleul Silvaniei $(35.86 \pm 0.04 \mathrm{mg} / \mathrm{L})$ in 2013 , followed by the same variety from Turulung Vii $(44.80 \pm 0.17$ $\mathrm{mg} / \mathrm{L}$ ) in 2013. The differences between variants were statistically assured ( $\mathrm{F}=1529.73, \mathrm{p} \leq 0.000)$ (Table 2). All the factors in the study influenced very significantly this character (Tab. 2).

In order to determine whether major wine quality parameters can influence the quality of wine and if they influence each other, correlations were performed to determine which of the parameters analyzed had greater influence. 
Tab.1. Qualitative characteristics of tested cultivars in studied areas

\begin{tabular}{|c|c|c|c|c|c|c|}
\hline Location & Years & $\begin{array}{l}\text { Alcohol } \\
\text { (\% vol.) }\end{array}$ & $\begin{array}{l}\text { Total acidity } \\
\left(\mathrm{g} / \mathrm{l}_{4} \mathrm{H}_{6} \mathrm{O}_{6}\right)\end{array}$ & $\begin{array}{l}\text { Volatile acidity } \\
\left(\mathrm{g} / \mathrm{l} \mathrm{CH}_{3} \mathrm{COOH}\right)\end{array}$ & $\mathrm{pH}$ & $\begin{array}{l}\text { Density } \\
\left(\mathrm{g} / \mathrm{cm}^{3}\right) \\
\end{array}$ \\
\hline Baia Mare & 2013 & $10.1 \pm 0.1 \mathrm{~h} \gamma$ & $7.15 \pm 0.04 \mathrm{j} \beta \gamma \delta$ & $0.59 \pm 0.04 \mathrm{ab} \alpha \beta$ & $3.3 \pm 0.1$ efg $\alpha \beta$ & $0.9954 \pm 0.0002 \operatorname{cdef} \beta$ \\
\hline & 2014 & $11.7 \pm 0.2 \operatorname{def} \beta$ & $7.26 \pm 0.03$ fgh $\beta \gamma$ & $0.44 \pm 0.06 \operatorname{cdefgh} \gamma \delta$ & $3.4 \pm 0.2$ abcd $\alpha$ & $0.9956 \pm 0.0004 \operatorname{cdef} \beta$ \\
\hline $\begin{array}{l}\text { Şimleul } \\
\text { Silvaniei }\end{array}$ & 2013 & $11.9 \pm 0.2 \mathrm{de} \beta$ & $7.36 \pm 0.02$ efgh $\beta$ & $0.41 \pm 0.02$ efghij $\gamma \delta$ & $3.3 \pm 0.3 \operatorname{cdefg} \alpha \beta$ & $0.9965 \pm 0.0002$ bc $\alpha$ \\
\hline & 2014 & $12.8 \pm 0.1 \mathrm{ab} \alpha$ & $6.92 \pm 0.05 \mathrm{k} \gamma \delta \varepsilon$ & $0.51 \pm 0.07 \mathrm{bcd} \beta \gamma$ & $3.4 \pm 0.3$ bcde $\alpha$ & $0.9970 \pm 0.0002 \mathrm{ab} \alpha$ \\
\hline $\begin{array}{c}\text { Turulung } \\
\text { Vii }\end{array}$ & 2013 & $11.6 \pm 0.2 \mathrm{bc} \beta$ & $7.76 \pm 0.16 \mathrm{ab} \alpha$ & $0.65 \pm 0.13$ a $\alpha$ & $3.2 \pm 0.1$ efg $\alpha \beta$ & $0.9954 \pm 0.0007 \operatorname{cdef} \beta$ \\
\hline \multirow{5}{*}{$\begin{array}{c}\text { 'Fetească } \\
\text { albă' }\end{array}$} & 2014 & $12.5 \pm 0.2$ ef $\alpha$ & $6.81 \pm 0.09 \mathrm{kl} \delta \varepsilon$ & $0.47 \pm 0.05 \operatorname{cdefg} \gamma \delta$ & $3.3 \pm 0.2$ cdefg $\alpha \beta$ & $0.9954 \pm 0.0004 \operatorname{cdef} \beta$ \\
\hline & 2013 & $11.5 \pm 0.3 \operatorname{def} \beta$ & $6.70 \pm 0.20 \mathrm{l} \varepsilon$ & $0.36 \pm 0.06$ hij $\delta$ & $3.2 \pm 0.1 \operatorname{defg} \alpha \beta$ & $0.9936 \pm 0.0009 \mathrm{~h} \gamma$ \\
\hline & 2014 & $12.4 \pm 0.6$ bc $\alpha$ & $7.23 \pm 0.51$ hi $\beta \gamma$ & $0.39 \pm 0.02$ efghij $\delta$ & $3.1 \pm 0.1 \mathrm{~g} \beta$ & $0.9952 \pm 0.0004 \operatorname{def} \beta$ \\
\hline & 2013 & $9.8 \pm 0.4 \mathrm{~h} \varepsilon$ & $7.93 \pm 0.09$ a $\alpha$ & $0.36 \pm 0.05$ hij $\gamma \delta$ & $3.5 \pm 0.1 \mathrm{abc} \alpha \beta$ & $0.9951 \pm 0.0001 \operatorname{def} \alpha$ \\
\hline & 2014 & $11.3 \pm 0.2 \mathrm{f} \beta \gamma$ & $7.74 \pm 0.10 \mathrm{abc} \beta$ & $0.38 \pm 0.03$ ghij $\beta \gamma \delta$ & $3.3 \pm 0.2 \operatorname{defg} \gamma$ & $0.9956 \pm 0.0005 \operatorname{cdef} \alpha$ \\
\hline $\begin{array}{l}\text { Şimleul } \\
\text { Silvaniei } \\
\end{array}$ & 2013 & $10.8 \pm 0.1 \mathrm{~g} \gamma \delta$ & $7.62 \pm 0.03$ bcd $\beta \gamma$ & $0.47 \pm 0.01$ cdefg $\alpha \beta$ & $3.6 \pm 0.2 \mathrm{ab} \alpha \beta$ & $0.9961 \pm 0.0002$ bcde $\alpha$ \\
\hline \multirow[t]{3}{*}{$\begin{array}{c}\text { ‘Fetească } \\
\text { regală’ }\end{array}$} & 2014 & $11.8 \pm 0.3 \operatorname{def} \beta$ & $7.45 \pm 0.13 \mathrm{ab} \beta$ & $0.49 \pm 0.07$ cde $\alpha$ & $3.5 \pm 0.1 \mathrm{bcdef} \beta \gamma$ & $0.9958 \pm 0.0012$ cde $\alpha$ \\
\hline & 2013 & $10.6 \pm 0.1 \mathrm{~g} \delta$ & $7.62 \pm 0.14$ bcd $\beta \gamma$ & $0.44 \pm 0.06$ cdefgh $\alpha \beta \gamma$ & $3.7 \pm 0.2$ a $\alpha$ & $0.9950 \pm 0.0011 \operatorname{def} \alpha$ \\
\hline & 2014 & $11.5 \pm 0.3 \operatorname{def} \beta$ & $7.49 \pm 0.04 \operatorname{cdef} \gamma$ & $0.51 \pm 0.07 \mathrm{bcd} \alpha$ & $3.4 \pm 0.1$ bcdef $\beta \gamma$ & $0.9947 \pm 0.0005$ efg $\alpha \beta$ \\
\hline \multirow[t]{2}{*}{ Blaj } & 2013 & $11.7 \pm 0.1 \operatorname{def} \beta$ & $5.50 \pm 0.07 \mathrm{n} \varepsilon$ & $0.38 \pm 0.07$ ghij $\beta \gamma \delta$ & $3.4 \pm 0.3 \mathrm{bcdef} \beta \gamma$ & $0.9919 \pm 0.0004 \mathrm{i} \gamma$ \\
\hline & 2014 & $12.5 \pm 0.5 \mathrm{bc} \alpha$ & $7.18 \pm 0.06$ hi $\delta$ & $0.33 \pm 0.02$ ij $\delta$ & $3.5 \pm 0.1 \mathrm{abc} \alpha \beta$ & $0.9935 \pm 0.0013 \mathrm{~h} \beta$ \\
\hline \multirow[t]{2}{*}{ Baia Mare } & 2013 & $10.5 \pm 0.1 \mathrm{~g} \varepsilon$ & $7.32 \pm 0.05$ efgh $\beta \gamma$ & $0.61 \pm 0.04 \mathrm{ab} \alpha$ & $3.2 \pm 0.2$ efg $\alpha$ & $0.9954 \pm 0.0002$ cdef $\gamma$ \\
\hline & 2014 & $11.8 \pm 0.4 \operatorname{def} \gamma \delta$ & $7.42 \pm 0.05 \operatorname{defg} \alpha \beta$ & $0.52 \pm 0.12 \mathrm{bcd} \beta \gamma$ & $3.2 \pm 0.2 \mathrm{efg} \alpha$ & $0.9952 \pm 0.0001$ def $\gamma$ \\
\hline $\begin{array}{l}\text { Şimleul } \\
\text { Silvaniei } \\
\end{array}$ & 2013 & $11.7 \pm 0.3$ ef $\delta$ & $6.91 \pm 0.04 \mathrm{k} \delta$ & $0.53 \pm 0.02$ bc $\alpha \beta$ & $3.3 \pm 0.1 \operatorname{cdefg} \alpha$ & $0.9980 \pm 0.0001$ a $\alpha$ \\
\hline \multirow[t]{3}{*}{$\begin{array}{c}\text { 'Italian } \\
\text { Riesling' }\end{array}$} & 2014 & $12.2 \pm 0.1 \mathrm{~cd} \beta \gamma$ & $7.21 \pm 0.05$ hi $\gamma$ & $0.48 \pm 0.09 \operatorname{cdef} \beta \gamma \delta$ & $3.2 \pm 0.1 \mathrm{fg} \alpha$ & $0.9971 \pm 0.0002 \mathrm{ab} \beta$ \\
\hline & 2013 & $11.3 \pm 0.2 \mathrm{f} \delta$ & $7.32 \pm 0.05$ efgh $\beta \gamma$ & $0.42 \pm 0.06$ defghi $\delta \varepsilon$ & $3.2 \pm 0.1$ efg $\alpha$ & $0.9979 \pm 0.0006$ а $\alpha \beta$ \\
\hline & 2014 & $12.4 \pm 0.3 \mathrm{bc} \beta$ & $7.56 \pm 0.07$ bcde $\alpha$ & $0.44 \pm 0.02$ cdefgh $\gamma \delta \varepsilon$ & $3.3 \pm 0.2$ cdefg $\alpha$ & $0.9970 \pm 0.0003 \mathrm{ab} \beta$ \\
\hline \multirow[t]{2}{*}{ Blaj } & 2013 & $11.4 \pm 0.5$ ef $\delta$ & $5.60 \pm 0.18 \mathrm{n} \delta$ & $0.38 \pm 0.03$ fghij $\varepsilon \zeta$ & $3.1 \pm 0.1 \mathrm{~g} \alpha$ & $0.9948 \pm 0.0009 \mathrm{fg} \gamma \delta$ \\
\hline & 2014 & $13.0 \pm 0.1$ a $\alpha$ & $6.08 \pm 0.05 \mathrm{~m} \varepsilon$ & $0.32 \pm 0.01 \mathrm{j} \zeta$ & $3.3 \pm 0.2 \operatorname{cdefg} \alpha$ & $0.9939 \pm 0.0008$ gh $\delta$ \\
\hline Sig. & & $\mathrm{p} \leq 0.000$ & $\mathrm{p} \leq 0.000$ & $\mathrm{p} \leq 0.000$ & $\mathrm{p} \leq 0.000$ & $\mathrm{p} \leq 0.000$ \\
\hline Location & & $* * *$ & $* * *$ & $* * *$ & $*$ & $* * *$ \\
\hline Variety & & $* * *$ & $* * *$ & $* *$ & $* * *$ & $* * *$ \\
\hline Years & & $* * *$ & *** & * & ns & ns \\
\hline Location x Variety & & $* * *$ & *** & $* * *$ & $*$ & $* * *$ \\
\hline Location $x$ Years & & $* *$ & $* * *$ & * & ns & $* *$ \\
\hline Variety $x$ Years & & ns & $* * *$ & ns & $* *$ & $* *$ \\
\hline $\begin{array}{c}\text { Location } x \text { Variety } x \\
\text { Years }\end{array}$ & & * & *** & $* * *$ & $* *$ & ns \\
\hline
\end{tabular}

$\overline{\text { Average value } \pm \text { standard deviation }(\mathrm{n}=3 \text { ). Different letters are significantly different for } \mathrm{P} \leq 0.05 \text { between varieties. The difference between any }}$ two values, followed by at least one common letter, is insignificant. Significance of area, variety, and interaction of these factors area $\mathrm{x}$ variety was tested for $\mathrm{P} \leq 0.05\left(^{*}\right), \mathrm{P} \leq 0.01\left(^{* *}\right)$, and $\mathrm{P} \leq 0.0001\left(^{* * *}\right)$. 
Tab. 2. Qualitative characteristics of tested cultivars in studied areas

\begin{tabular}{|c|c|c|c|c|c|c|}
\hline Location & Years & $\begin{array}{c}\text { Residual sugar } \\
(\mathrm{g} / \mathrm{L})\end{array}$ & $\begin{array}{l}\text { Dry extract } \\
(\mathrm{g} / \mathrm{L})\end{array}$ & $\begin{array}{c}\text { Non-reducing extract } \\
(\mathrm{g} / \mathrm{L})\end{array}$ & $\begin{array}{l}\text { Free } \mathrm{SO}_{2} \\
(\mathrm{~g} / \mathrm{L}) \\
\end{array}$ & $\begin{array}{l}\text { Total } \mathrm{SO}_{2} \\
(\mathrm{~g} / \mathrm{L}) \\
\end{array}$ \\
\hline Baia Mare & 2013 & $2.6 \pm 0.2 \mathrm{~cd} \beta$ & $19.6 \pm 0.1 \mathrm{gh} \varepsilon$ & $17.0 \pm 0.2$ hi $\delta$ & $19.32 \pm 0.05 \mathrm{~g} \delta$ & $143.36 \pm 0.10 \mathrm{~g} \varepsilon$ \\
\hline & 2014 & $2.2 \pm 0.1$ ef $\gamma$ & $23.4 \pm 0.7 \mathrm{~cd} \gamma$ & $21.1 \pm 1.4$ ef $\beta$ & $15.40 \pm 0.83$ i $\varepsilon$ & $147.97 \pm 0.87 \mathrm{f} \delta$ \\
\hline $\begin{array}{l}\text { Şimleul } \\
\text { Silvaniei }\end{array}$ & 2013 & $2.6 \pm 0.1 \mathrm{~d} \beta$ & $25.8 \pm 0.2 \mathrm{~b} \beta$ & $23.2 \pm 0.1$ de $\alpha$ & $5.12 \pm 0.03 \mathrm{~lm} \eta$ & $64.00 \pm 0.05$ о $\eta$ \\
\hline \multirow[t]{8}{*}{ Fetească albă } & 2014 & $2.5 \pm 0.3 \mathrm{~d} \beta$ & $28.4 \pm 1.4$ a $\alpha$ & $22.8 \pm 1.1 \mathrm{~d} \alpha \beta$ & $25.87 \pm 0.12 \mathrm{f} \gamma$ & $165.06 \pm 0.62 \mathrm{~d} \beta$ \\
\hline & 2013 & $1.8 \pm 0.1 \mathrm{~h} \delta$ & $23.2 \pm 0.4$ cde $\gamma$ & $21.4 \pm 0.7$ ef $\beta$ & $4.52 \pm 0.07 \mathrm{~m} \mathrm{\eta}$ & $52.52 \pm 0.08$ pr $\theta$ \\
\hline & 2014 & $3.1 \pm 0.3 \mathrm{~b} \alpha$ & $24.7 \pm 0.9$ bc $\beta \gamma$ & $21.6 \pm 0.3$ ef $\beta$ & $10.43 \pm 0.28 \mathrm{k} \zeta$ & $121.62 \pm 1.05 \mathrm{~lm} \zeta$ \\
\hline & 2013 & $0.9 \pm 0.1 \mathrm{j} \varepsilon$ & $20.1 \pm 1.6$ fgh $\delta \varepsilon$ & $19.0 \pm 0.9 \mathrm{~g} \gamma$ & $28.14 \pm 0.93$ e $\beta$ & $169.74 \pm 4.54 \mathrm{c} \alpha$ \\
\hline & 2014 & $1.0 \pm 0.1 \mathrm{j} \varepsilon$ & $21.5 \pm 0.9$ ef $\delta$ & $21.4 \pm 1.1$ ef $\beta$ & $35.91 \pm 1.45$ с $\alpha$ & $159.40 \pm 5.50$ e $\gamma$ \\
\hline & 2013 & $2.0 \pm 0.2 \operatorname{fgh} \delta$ & $18.5 \pm 0.2 \mathrm{~h} \delta$ & $16.5 \pm 0.3$ i $\delta$ & $6.48 \pm 0.071 \delta$ & $55.04 \pm 0.07 \mathrm{p} \zeta$ \\
\hline & 2014 & $1.9 \pm 0.2 \mathrm{gh} \delta$ & $22.8 \pm 2.4 \operatorname{cde} \alpha \beta$ & $20.7 \pm 0.9 \mathrm{f} \beta$ & $10.59 \pm 0.22 \mathrm{k} \gamma$ & $123.59 \pm 0.71 \mathrm{kl} \delta$ \\
\hline & 2013 & $1.9 \pm 0.2 \mathrm{gh} \delta$ & $22.7 \pm 0.3$ cde $\alpha \beta$ & $20.8 \pm 0.1$ ef $\beta$ & $4.56 \pm 0.03 \mathrm{~m} \varepsilon$ & $35.86 \pm 0.04 \mathrm{t} \theta$ \\
\hline \multirow[t]{3}{*}{$\begin{array}{c}\text { 'Fetească } \\
\text { regală' }\end{array}$} & 2014 & $2.5 \pm 0.1$ de $\beta$ & $24.2 \pm 0.9$ bc $\alpha$ & $21.9 \pm 0.4$ ef $\beta$ & $12.75 \pm 0.47 \mathrm{j} \gamma$ & $118.32 \pm 0.95 \mathrm{~m} \varepsilon$ \\
\hline & 2013 & $1.5 \pm 0.1$ i $\varepsilon$ & $20.1 \pm 0.1 \operatorname{fgh} \gamma \delta$ & $18.6 \pm 0.8 \mathrm{~g} \gamma$ & $3.56 \pm 0.05 \mathrm{~m} \varepsilon$ & $44.80 \pm 0.17 \mathrm{~s} \eta$ \\
\hline & 2014 & $2.9 \pm 0.3 \mathrm{bc} \beta$ & $23.7 \pm 1.9 \mathrm{~cd} \alpha \beta$ & $21.1 \pm 1.3$ ef $\beta$ & $11.31 \pm 1.01 \mathrm{jk} \gamma$ & $130.60 \pm 1.44 \mathrm{j} \gamma$ \\
\hline \multirow[t]{2}{*}{ Blaj } & 2013 & $4.9 \pm 0.1$ a $\alpha$ & $22.6 \pm 1.0$ cde $\alpha \beta$ & $17.5 \pm 1.3$ ghi $\gamma \delta$ & $31.61 \pm 2.91 \mathrm{~d} \beta$ & $186.76 \pm 3.83$ a $\alpha$ \\
\hline & 2014 & $1.5 \pm 0.2$ i $\varepsilon$ & $21.8 \pm 1.0$ def $\beta \gamma$ & $23.4 \pm 1.0 \mathrm{~cd} \alpha$ & $47.73 \pm 1.84 \mathrm{~b} \alpha$ & $136.89 \pm 4.35$ i $\beta$ \\
\hline \multirow[t]{2}{*}{ Baia Mare } & 2013 & $2.2 \pm 0.1$ ef $\beta$ & $20.5 \pm 0.3 \mathrm{fg} \gamma$ & $18.3 \pm 0.1 \mathrm{gh} \delta$ & $12.56 \pm 0.10 \mathrm{j} \delta$ & $93.44 \pm 0.05 \mathrm{n} \zeta$ \\
\hline & 2014 & $2.0 \pm 0.3 \operatorname{fgh} \beta$ & $23.4 \pm 0.6$ cde $\beta$ & $21.4 \pm 0.3$ ef $\gamma$ & $11.63 \pm 0.70 \mathrm{jk} \delta \varepsilon$ & $125.33 \pm 3.62 \mathrm{k} \delta$ \\
\hline $\begin{array}{l}\text { Şimleul } \\
\text { Silvaniei } \\
\end{array}$ & 2013 & $2.2 \pm 0.1 \mathrm{fg} \beta$ & $28.6 \pm 0.3$ a $\alpha$ & $26.4 \pm 0.3$ a $\alpha$ & $5.28 \pm 0.01 \mathrm{~lm} \zeta$ & $52.48 \pm 0.18 \mathrm{pr} \eta$ \\
\hline \multirow[t]{3}{*}{$\begin{array}{r}\text { 'Italian } \\
\text { Riesling' }\end{array}$} & 2014 & $2.8 \pm 0.8 \mathrm{~b} \alpha$ & $27.9 \pm 0.7$ a $\alpha$ & $24.6 \pm 0.4$ bc $\beta$ & $14.49 \pm 0.36 \mathrm{i} \gamma$ & $137.76 \pm 0.15 \mathrm{i} \gamma$ \\
\hline & 2013 & $2.1 \pm 0.2 \mathrm{fg} \beta$ & $27.6 \pm 1.2$ a $\alpha$ & $25.5 \pm 1.1 \mathrm{ab} \alpha \beta$ & $5.28 \pm 0.04 \mathrm{~lm} \zeta$ & $51.24 \pm 0.11 \mathrm{r} \eta$ \\
\hline & 2014 & $3.1 \pm 0.2 \mathrm{~b} \alpha$ & $28.7 \pm 0.8$ a $\alpha$ & $25.4 \pm 0.6 \mathrm{ab} \alpha \beta$ & $10.55 \pm 0.16 \mathrm{k} \varepsilon$ & $121.22 \pm 0.31 \mathrm{~lm} \varepsilon$ \\
\hline \multirow[t]{2}{*}{ Blaj } & 2013 & $1.0 \pm 0.2 \mathrm{j} \gamma$ & $18.7 \pm 0.9 \mathrm{~h} \delta$ & $17.7 \pm 0.9$ ghi $\delta$ & $26.11 \pm 1.95 \mathrm{f} \beta$ & $176.17 \pm 1.52 \mathrm{~b} \alpha$ \\
\hline & 2014 & $1.1 \pm 0.1 \mathrm{j} \gamma$ & $19.5 \pm 1.1 \mathrm{gh} \gamma \delta$ & $21.0 \pm 1.5$ ef $\gamma$ & $50.93 \pm 1.94$ a $\alpha$ & $170.65 \pm 3.47 \mathrm{c} \beta$ \\
\hline Sig. & & $\mathrm{p} \leq 0.000$ & $\mathrm{p} \leq 0.000$ & $\mathrm{p} \leq 0.000$ & $\mathrm{p} \leq 0.000$ & $\mathrm{p} \leq 0.000$ \\
\hline Location & & $* * *$ & $* * *$ & $* * *$ & $* * *$ & $* * *$ \\
\hline Variety & & $* * *$ & $* * *$ & $* * *$ & $* * *$ & $* * *$ \\
\hline Years & & $*$ & $* * *$ & $* * *$ & $* * *$ & $* * *$ \\
\hline Location $x$ Variety & & $* * *$ & $* * *$ & $* * *$ & $* * *$ & $* * *$ \\
\hline Location $x$ Years & & $* * *$ & $* * *$ & $* * *$ & $* * *$ & $* * *$ \\
\hline Variety $x$ Years & & $* * *$ & ns & $* * *$ & $* *$ & $* *$ \\
\hline Location $x$ Variety $x$ Years & & $* * *$ & $*$ & $*$ & $* * *$ & $* * *$ \\
\hline
\end{tabular}

Average value \pm standard deviation $(\mathrm{n}=3$ ). Different letters are significantly different for $\mathrm{P} \leq 0.05$ between varieties. The difference between any two values, followed by at least one common letter, is insignificant. Significance of area, variety, and interaction of these factors area $\mathrm{x}$ variety was tested for $\mathrm{P} \leq 0.05(*), \mathrm{P} \leq 0.01(* *)$, and $\mathrm{P} \leq 0.0001\left(^{* * *}\right)$. 
Tab. 3. Pearson correlation between the physicochemical analyses of wine

\begin{tabular}{|c|c|c|c|c|c|c|c|c|c|c|}
\hline & $\begin{array}{l}\text { Alcohol } \\
\text { (\% vol.) }\end{array}$ & $\begin{array}{c}\text { Total } \\
\text { Acidity } \\
(\mathrm{g} / \mathrm{L} \\
\left.\mathrm{C}_{2} \mathrm{H}_{2} \mathrm{O}_{6}\right) \\
\end{array}$ & $\begin{array}{c}\text { Volatile } \\
\text { acidity } \\
(\mathrm{g} / \mathrm{L} \\
\left.\mathrm{CH}_{3} \mathrm{COOH}\right)\end{array}$ & $\mathrm{pH}$ & $\begin{array}{l}\text { Density } \\
\left(\mathrm{g} / \mathrm{cm}^{3}\right)\end{array}$ & $\begin{array}{c}\text { Residual } \\
\text { sugar }(\mathrm{g} / \mathrm{L})\end{array}$ & $\begin{array}{c}\text { Dry } \\
\text { Extract } \\
\text { (g/L) }\end{array}$ & $\begin{array}{l}\text { Non- } \\
\text { reducing } \\
\text { Extract } \\
(\mathrm{g} / \mathrm{L})\end{array}$ & $\begin{array}{c}\text { Fee } \mathrm{SO}_{2} \\
(\mathrm{~g} / \mathrm{L})\end{array}$ & $\begin{array}{c}\text { Total SO } \\
\text { (g/L) }\end{array}$ \\
\hline Alcohol (\% vol.) & 1.000 & & & & & & & & & \\
\hline Total Acidity & $-0.321^{* *}$ & 1.000 & & & & & & & & \\
\hline Volatile acidity & -0.218 & $0.292 *$ & 1.000 & & & & & & & \\
\hline $\mathrm{pH}$ & -0.154 & 0.231 & -0.155 & 1.000 & & & & & & \\
\hline Density & -0.033 & $0.466^{* *}$ & $0.291^{*}$ & -0.135 & 1.000 & & & & & \\
\hline Residual sugar & 0.043 & -0.117 & 0.216 & 0.100 & 0.018 & 1.000 & & & & \\
\hline Dry Extract & $0.423^{* *}$ & 0.186 & 0.218 & -0.034 & $0.654^{* *}$ & $0.428^{* *}$ & 1.000 & & & \\
\hline Non-reducing Extract & $0.534^{* *}$ & 0.220 & 0.074 & -0.131 & $0.599^{* *}$ & 0.066 & $0.792^{* *}$ & 1.000 & & \\
\hline Fee $\mathrm{SO}_{2}$ & $0.514^{* *}$ & $-0.591^{* *}$ & $-0.464^{* *}$ & -0.083 & $-0.575^{* *}$ & $-0.232^{*}$ & $-0.335^{* *}$ & -0.125 & 1.000 & \\
\hline Total $\mathrm{SO}_{2}$ & $0.480^{* *}$ & $-0.633^{* *}$ & $-0.286^{*}$ & $-0.251^{*}$ & $-0.487^{* *}$ & 0.084 & -0.182 & -0.217 & $0.753^{* *}$ & 1.000 \\
\hline
\end{tabular}

Regarding the Pearson correlation coefficients (Tab. 3), it can be stated that a large number of positive and negative correlations was obtained between various parameters: alcohol and total acidity $\left(-0.321^{* *}\right)$; free $\mathrm{SO}_{2}$ and total acidity $\left(-0.633^{* *}\right)$; dry extract and density $\left(0.654^{* *}\right)$; non-reducing extract and dry extract $\left(0.792^{* *}\right)$; total $\mathrm{SO}_{2}$ and free $\mathrm{SO}_{2}\left(0753^{* *}\right)$. As it can be seen, the correlation coefficients obtained indicate the existence of close links between wine quality parameters.

Regarding to alcohol degree the values obtained are comparable those obtained by Ghiță et al., 2013 (11.2 \% vol. Arad-'Fetească regală'); Postolache et al., 2012 (12.0 \% vol. Dealu Bujorului-'Fetească regală'; $11.0 \%$ vol. Dealu Bujorului-'Fetească albă'); Rotaru et al., 2010 (11.4 $\%$ vol. Cotnari-'Fetească albă'). Total acidity values obtained are specific vine varieties investigated also this values are in the normal rage. Wine must have a minimum of $4.5 \mathrm{~g} / \mathrm{l} \mathrm{C}_{4} \mathrm{H}_{6} \mathrm{O}_{6}$ (Țârdea et al., 2007). The values obtained for residual sugar are comparable with those obtained by Iliescu et al., 2008 (2.3) in vineyard Tarnave.

Also the values obtained for dry extract are comparable whit values obtained by Postolache et al., 2012 (23.4 g/L Dealu Bujorului-'Fetească albă'); Rotaru et al., 2010 (22.7 g/L Cotnari'Fetească albă';); Odăgeriu et al., 2012 (22.9 g/L Huși-'Fetească albă'); Iliescu et al., 2008 (20.0 g/L Tarnave-'Riesling italian'); Iliescu et al., 2008 (19.6 g/L Tarnave-'Fetească regală'.
Regarding the free $\mathrm{SO}_{2}(50.93 \mathrm{~g} / \mathrm{L}$ obtained by 'Riesling italian' varieties cultivated in Blaj location) and total $\mathrm{SO}_{2}$ (186.76 g/L obtained by 'Fetească regală' varieties cultivated in same location) the values of wine are below the maximum allowed by law.

\section{CONCLUSIONS}

The quality of wine obtained from 'Fetească albă', 'Fetească regală' and 'Italian Riesling', in the years 2013 and 2014, in the four areas, was particularly influenced by the balance between alcoholic strength, acidity and residual sugar.

The wine with the highest alcoholic strength was obtained in Blaj (Târnave Vineyard) and Şimleul Silvaniei (Silvaniei Vineyard), wellestablished winegrowing areas, where due to heliothermal resources, the varieties accumulate large amounts of sugar and top-quality wines may be obtained.

In Baia Mare vines are grown mostly in an amateuristic way, and in some years current table wine can be obtained with an alcoholic strength below $11 \%$ vol.

The acidity is negatively correlated with the accumulation of sugar or alcoholic strength. The acidity was higher in Baia Mare and Turulung Vii and lower in Şimleul Silvaniei and Blaj, this is why in the last two areas harvesting the grapes must be done earlier in order to obtain balanced wines.

For all varieties, in all locations and in both years, the amount of residual sugar has lower values than $4 \mathrm{~g} / \mathrm{L}$ and the wines are dry. In 
Şimleul Silvaniei and Turulung Vii there have been obtained the most extractive wines, compared with those from Blaj and Baia Mare.

By sulphitation of must and wine, in order to clarify and conserve them with higher amounts of $\mathrm{SO}_{2}$ in consecrated areas, but within the limits allowed by law, it has been noted that wine has higher quantities of $\mathrm{SO}_{2}$ than on private properties from unacknowledged areas.

Comparing the results regarding the total $\mathrm{SO}_{2}$ content with the legislation, it can be concluded that all produced wines have a much lower content than the one required by law.

Acknowledgments. This paper was published under the frame of European Social Fund, Human Resources Development Operational Programme 2007-2013, project no. POSDRU/159/1.5/S/132765.

\section{REFERENCES}

1. Bora FD, Pop TI, Bunea CI, Urcan DE, Babeș A, Cozmuta ML, Cozmuta MA, Pop N (2014). Influence of ecoclimatic and ecopedological conditions on quality of white wine grape varieties from North-West of Romania. Bulletin UASVM Horticulture 71(2): 218-225.

2. Dalipi R, Borgese L, Zacco A, Tsuji K, Sangiorgi E, Piro R, Bontempi E, Depero LE (2015). Determination of trace elements in Italian wines by means of total reflection X-ray fluorescence spectroscopy. Intern J Environ Anal Chem 95(1):1-11.

3. Değirmenci Karataș D, Aydin F, Aydin I, Karataș H (2015). Elemental composition of red wines in Southeast Turkey. Czech J. Food Sci 33:228-236.

4. Donici A, Simion C, Enache V (2013). Research on the behavior of some varieties of vines in the vineyard area of Dealu Bujorului. Scientific Papers Seria Horticulture UASVM Iași 56(1):285-290.

5. Fernandez C (1988). The importance of metallic elements in wine. A literature survey. Zeitschrift für LebensmittelUntersuchunf und- Forschung 186:295-300.

6. Ghiță AG, Dobrei A. Mălăescu M. Drăgunescu A, Ciucur A (2013). Research on the potential quality and quality of some varieties of wine under the conditions in Paulian village, Arad Country. Journal of Horticulture, Forestry and Biotechnology 17(1):108-111.

7. Gonçalves da Silva AM, Pavan MA, Muniz AS, Tonin TA, Pelizer T (2008). Nutrient availability in the soil and its absorption, transport, and redistribution in vines. Communications in Soil Science and Plant Analysis 39:1507-1516

8. Iliescu M, Ranca A, Farago M, Moldovan SD, Bolos P (2008). The quality of the viticultural environment in the
Tarnave and Murfatlar vineyards, Romania. In: Proc. Pf the $31^{\text {st }}$ World Congress of Vine and Wine and $6^{\text {st }}$ General Assembly of the O.I.V. June, Verona, Italy.

9. Katalinic V, Milos M, Modum D, Music I, Boban M (2004). Antioxidant effectiveness of selected wines in comparison with (+)-catechin. Food Chemistry 86:593:600.

10. Lădaru GR, Beciu S, Vlad IM (2014). Analysis on the evolution of surfaces under vine in Romania (20032013). Scientific Papers Series Management, Economic Engineering in Agriculture and Rural Development 14(4) 153-156.

11. Marini F, Bucci R, Magri AL, Magri AD (2006). Authentication of Italian CDO wines by class-modeling techniques. Chemometrics and Intelligent Laboratory Systems 84:164-171.

12. Monaci F, Bargagli R, Focardi S (2003). Element concentration in Chianti Classico appellation wines. Journal of Trace Elements in Medicine and Biology 17:4550

13. Nilsson M, Duarte IF, Almeida C, Delgadillo I, Goodfellow BJ, Gil AM, Moris GA (2004). High-resolution NMR and diffusion-ordered spectroscopy of port wine. Journal of Agricultural and Food Chemistry 52:3736-3743.

14. Núñez M, Pena RM, Herrero C, García-Martín S (2000). Analysis of some metals in wine by means of capillary electrophoresis. Application to the differentiation of Ribeira Sacra Spanish red wine. Analysis 28:432-437.

15. Odăgeriu GH, Zamfir CI, Crețu C, Cotea V (2012). Compositional aspects of quality wines produced in Averești wine growing of Huși vineyard, harvest of 2011. Lucrări Științifice 55, seria Agronomie.

16. Rotaru L, Filipov F, Mustea M, Soleru V (2010). Influence of some " Terroir Viticole" foctors on quality of grape. Not. Bot. Agrobot 38(1):176:181.

17. Roig B, Thomas $O$ (2003). UV monitoring of sugars during wine making. Carbohydrate Research 338:79-83.

18. Voica C, Dehelean A, Pamula A (2009). Method validation for determination of heavy metals in wine and slightly beverages by ICP-MS. J of Physics: Conference Series 182(1):1-5

19. Tabaranu G, Enache V, Donici A (2014). Study on the vulnerability vineyard ecosystem detrimental impact of competition and antagonistic organisms of vineyard plantations Dealu Bujorului. Conferința Științifică Internațională, Universitatea de Stat "Al. Russo" Republica Moldova 20:287-298.

20. Țârdea C (2007). Chemistry and wine analysis. Publisher "Ion Ionescu de la Brad", Iași, România.

21. Tudorache A, Pircalabu L., Porumb R, Tomoiaga L, Iliescu M, Enache V, Simion C, Dorina D, Petrescu A, Ghica M (2013). Description des climats dans les centres viticoles principaux de Roumanie. OIV 86:45-58.

22. Postolache E, Ciubucă A, Bîrliga N, Enache V (2013). The maturation on the grapes in the conditions of excessive drought of 2012 in the vineyard Dealu Bujorului. Lucrări științifice 56(1):301-306. 